\title{
20 Fabrication of Mesoscopic Devices Using Atomic Force Microscopic Electric Field Induced Oxidation
}

\author{
F. K. Lee, G. H. Wen, X. X. Zhang and O. K. C. Tsui* \\ Institute of Nano Science and Technology and Physics \\ Department, Hong Kong University of Science and \\ Technology, Clear Water Bay, Kowloon, Hong Kong
}

\section{INTRODUCTION}

We demonstrate the fabrication of mesoscopic devices on aluminum thin film by using atomic force microscopic (AFM) electric field induced oxidation together with selective wet etching. The device structure being demonstrated is a percolating network consisting of conducting dots (70 $\mathrm{nm}$ in diameter) randomly distributed within an area of $1 \times 1 \mathrm{\mu m}^{2}$. Details on how to fabricate the network structure and the making of electrical contacts to the device will be focused upon. Good agreement between the temperature-dependent resistivity and Hall coefficient measurements of an aluminum control sample we made and those reported in the literature for bulk aluminum warrants reliability of our sample fabrication technique.

\section{BACKGROUND}

While the critical dimension to increase the circuit density for faster microprocessors and high-density memories approaches nanometer scale, the conventional photolithography reaches its resolution limit due to far field diffraction effects. The corresponding resolution limit is of the order of the wavelength of the radiation used in the photo-exposure, i.e. about $200 \mathrm{~nm}$. Other techniques such as high-resolution electron beam lithography and scanning probe lithography have been developed to push the limit further to $10 \mathrm{~nm}$. Amongst them, atomic force microscope (AFM) emerges one very promising tool to perform the art in light of its versatility. It can pattern resist materials by lowenergy electron beam exposure, which avoids the proximity problem pertaining to electron beam lithography (Ishibashi et al., 1999). It also can pattern semiconductors and metals by electric field induced local oxidation (FILO) (Tsau et al., 1994, Snow et al., 1996) or mechanical scratching on multi-layer systems.

\footnotetext{
* Corresponding author. Email address: phtsui@ust.hk
} 
(Bouchiat and Esteve, 1996, Hu et al., 1998) Unlike scanning tunneling microscopes, AFM has the advantage of decoupling the exposure mechanism from the feedback control that governs the movement of the probe tip. This feature enables the AFM to work on insulating as well as conducting samples. In the past decade, many research studies had been focused on using AFM FILO for the fabrication of nanostructures (Dagata, 1995, Shirakashi, et al., 1996, Fontaine et al., 1998, Gwo et al., 1999, Chien et al., 2000) in semiconductors and metals since these materials and their corresponding oxides are frequently used in integrated circuits. By using the AFM FIL_O technique, many different kinds of sophisticated devices have been successfully rade. Just to name a few, Campbell et al. (1995) fabricated a side-gated silicon field effect transistor with critical features as small as $30 \mathrm{~nm}$; Minne et al. (1995) fabricated a metal oxide semiconductor field effect transistor (MOSFET) on silicon with an effective channel length of $0.1 \mu \mathrm{m}$; Itatani et al. (1996) fabricated ultrafast metalsemiconductor-metal photoconductive switches.

In this study, we will focus on the fabrication of aluminum nanostructures by AFM FILO. Aluminum is widely used as an interconnect layer for integrated circuits. But insofar, attempts had only been made in making simple structures such as parallel lines with the AFM method on this material (Boisen et al., 1998). In the report, the feasibility of AFM FILO to make arbitrary, pre-designed structures, which in this case consists of a random array of dots, on aluminum will be demonstrated. We then verify the reliability of our fabrication process by electrical measurements.

\section{EXPERIMENT}

The field-induced oxidation and sample characterization were carried out in a model SPN3800 AFM from Seiko Instruments (Chiba, Japan) operated under

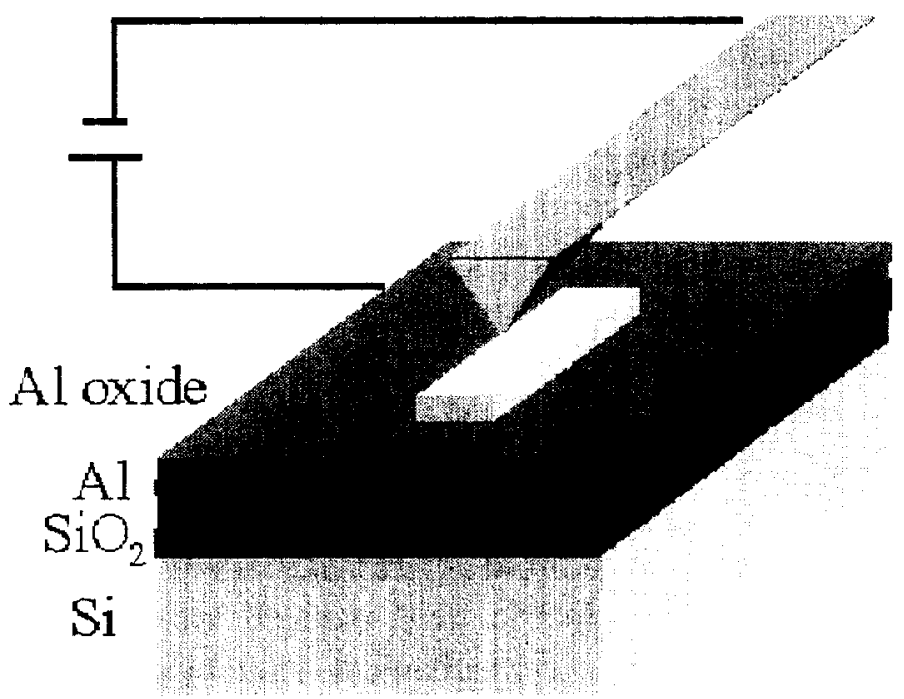

Figure 1 Schematic diagram of the setup for AFM electric field induced oxidation. 
ambient conditions. Platinum coated silicon probe tips were used in contact mode. In performing the field-induced oxidation, a positive biased voltage was applied to the sample surface with the AFM probe tip connected to the ground. A schematic diagram showing the setup is shown in Figure 1.

\subsection{AFM Lithography on Aluminum Films}

Aluminum thin films, of typical thickness $\sim 15 \mathrm{~nm}$, were deposited either by thermal evaporation or sputtering on $\mathrm{Si}(100)$ substrates covered with a $100 \mathrm{~nm}$ thick thermal silicon oxide. To fabricate the silicon oxide over-layer, a 4-inch n-type $\mathrm{Si}(100)$ wafer with resistivity $4-7 \Omega \mathrm{cm}$ was first cleaned in a mixture of $\mathrm{H}_{2} \mathrm{SO}_{4}$ and $\mathrm{H}_{2} \mathrm{O}_{2}$ in $10: 1$ ratio at $120^{\circ} \mathrm{C}$ for $10 \mathrm{mins}$. Then the thermal oxide was grown by passing a stream of water vapor through the cleaned wafer, which was kept at $1000{ }^{\circ} \mathrm{C}$ for 9 mins. $40 \mathrm{~s}$. To pattern the Al film, the AFM was programmed to send out voltage pulses of $5 \mathrm{~V}$ (height) and $0.6 \mathrm{~s}$ (width) to the probe tip at points on the sample where the dots were intended. Upon patterning by local oxidation, the $\mathrm{Al}$ film was dipped into a solution of phosphoric acid in which regions of the film not covered under the oxide were etched away. A random dot pattern in a $15 \times 15$ array thus fabricated on a $15 \mathrm{~nm}$ thick $\mathrm{Al}$ film is displayed in Figure 2.

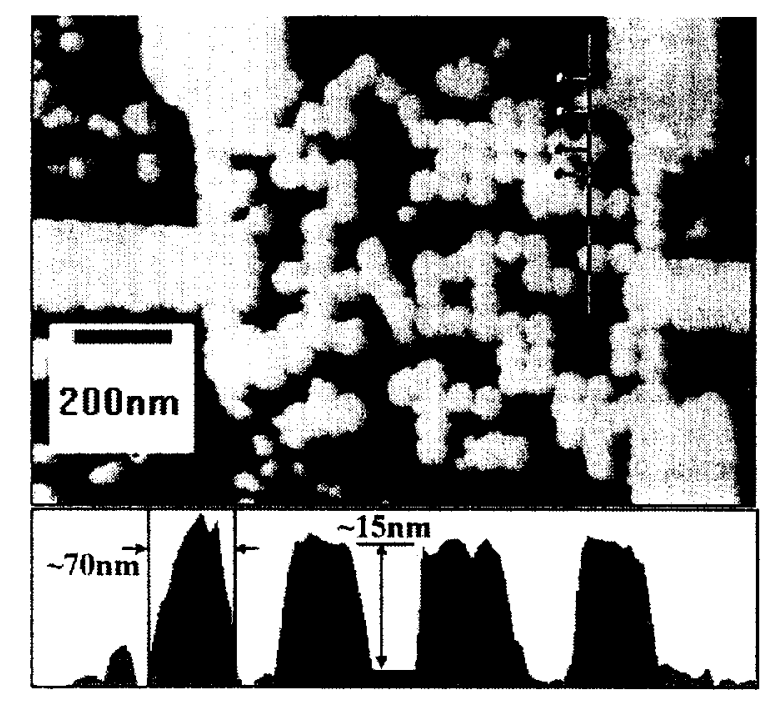

Figure 2 (Upper panel) AFM topographical image of a $15 \times 15$ dot pattern on an aluminum thin film fabricated by AFM electric field induced oxidation followed by selective wet etching. (Lower panel) Cross-sectional profile of the nanostructure along the horizontal line drawn across the image in the upper panel.

As seen, the width of the dots is about $70 \mathrm{~nm}$ on average with height $15 \mathrm{~nm}$. It is noteworthy that a smooth starting film with roughness less than $1 \mathrm{~nm}$ is crucial to the success of the AFM oxidation technique. The typical height of an AFM induced oxide feature is only a few nanometers. For the oxide pattern to be recognizable, which is necessary for the evaluation for the quality of the written feature, the film roughness 
must therefore be of sub-nanometer scale. To produce Al films with the desired smoothness, the films were made under high deposition rates $(>\sim 2 \mathrm{~nm} / \mathrm{s})$, which had a tendency to form nucleation centers at a higher density and metal grains of smaller sizes. The average roughness of $\mathrm{Al}$ film we made is $\sim 0.5 \mathrm{~nm}$.

\subsection{Fabrication of the Electrical Leads}

In order to be able to make electrical measurements on a nanostructure (which are about $1 \times 1 \mu \mathrm{m}^{2}$ for the ones we made), electrical leads must be fabricated to connect the structure to the electrodes of the measuring probe. Macroscopic gold contact pads (with lateral dimension $\sim 0.5 \mathrm{~mm}$ ) that converge toward and terminate near a common centering point (Figure 3(a)) were first fabricated on a thermal oxide covered Si substrate by photolithography followed by lift-off. A uniform film of $\mathrm{Al}$ was then deposited on top. At the converging point of the contact pads, the desired pattern was drawn by AFM field-induced oxidation as detailed above. Then electrical leads were drawn to connect the pattern structure to the nearby contact pads (Figure 3(b)).

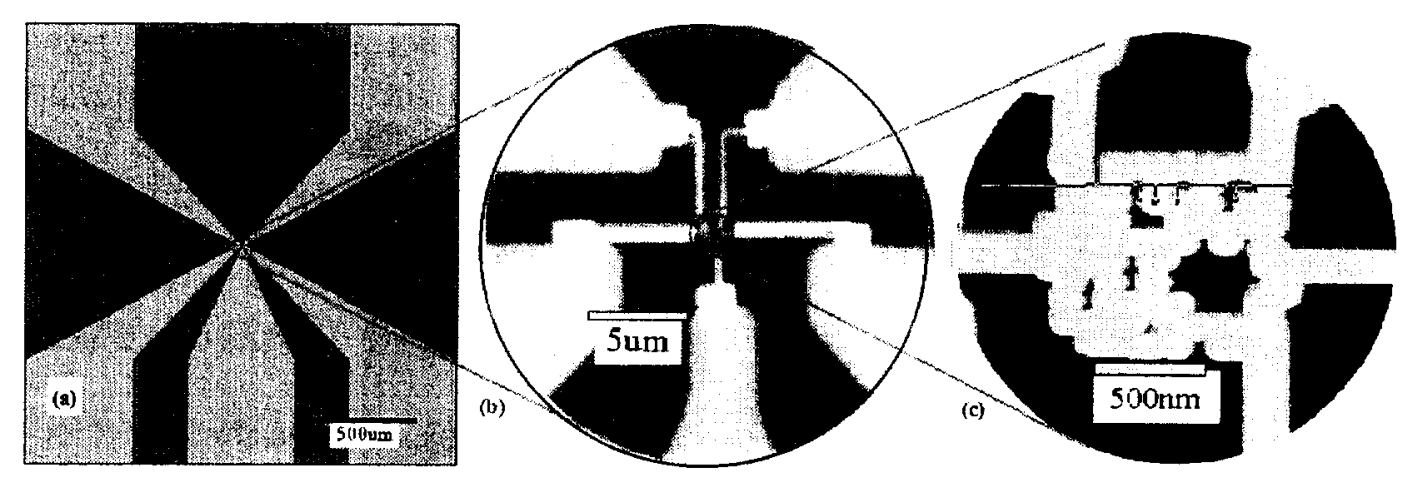

Figure 3 (a) Macroscopic contact pads of chromium/gold fabricated by photolithography and lift-off, (b) optical micrograph illustrating a centering aluminum dot pattern with connections to the nearby contact pads (c) AFM topographical image of the aluminum dot pattern.

Upon selective etching in phosphoric acid, the sample was ready for connection to an external measurement device. It is imperative that no soldering is involved at any stage of the wiring. We found that the nanostructure samples could be easily destroyed by the heat come out of a heated soldering iron even when the iron was held at a normal working distance about $20 \mathrm{~cm}$ from the sample. We used an interfacing connector of the plug-in type to make the connection. With a wire bonder, we first connected the contact pads on the sample to the connector's electrodes. With this, the connector could be easily fitted mechanically to a mating connector that was pre-mounted onto the measuring device. By this way, no soldering is involved in the wiring. 


\subsection{Results from Electrical Measurements}

Electrical measurements had been carried out on both the control sample and two samples containing the dot pattern with different metal fractions, $f=59 \%$ and $49 \%$, respectively. The control sample (i.e, one in which the sample area was plain) is of area $1 \times 1 \mu \mathrm{m}^{2}$ and thickness $22 \mathrm{~nm}$. The two dot pattern samples, also are of area $1 \times 1 \mu \mathrm{m}^{2}$, have thickness $\sim 16 \mathrm{~nm}$. Results from the resistivity $(\rho$ ) versus temperature $(T)$ measurements are displayed in Figure 4 . As seen, from $T=300 \mathrm{~K}$, $\rho$ of all the samples decrease linearly with decreasing $T$, demonstrating the metallic behavior. Near $\sim 50 \mathrm{~K}$, the curves come to a plateau, suggesting that defect scattering may have taken over to be the dominating mechanism. A similar $\rho-T$ behavior had also been found in an as-deposited bulk Al film similarly prepared by thermal evaporation (data not shown).

Moreover, the decrease of temperature coefficient of resistivity with decreasing metal fraction is consistent with the earlier study by Zhang et al. on co-sputtered $\mathrm{Cu} / \mathrm{SiO}_{2}$ composite films wherein the volume fraction of $\mathrm{Cu}$ was varied in the range $0.45 \leq f \leq 0.8$ (Zhang et al., 2000). In Figure 5, we show the result from a Hall resistance versus magnetic field $(H)$ measurement obtained at room temperature. The Hall coefficient, deduced from the slope of the curve, is $5.1 \times 10^{-11} \mathrm{~m}^{3} / \mathrm{C}$, which agrees quite wel? with the literature value reported for bulk aluminum $\left(=3.0 \times 10^{-11} \mathrm{~m}^{3} / \mathrm{C}\right.$ ) (Stokes, 1987). These results are important in assuring the reliability of the process we have devised to fabricate nanostructures and the connecting electrical leads with AFM lithography.

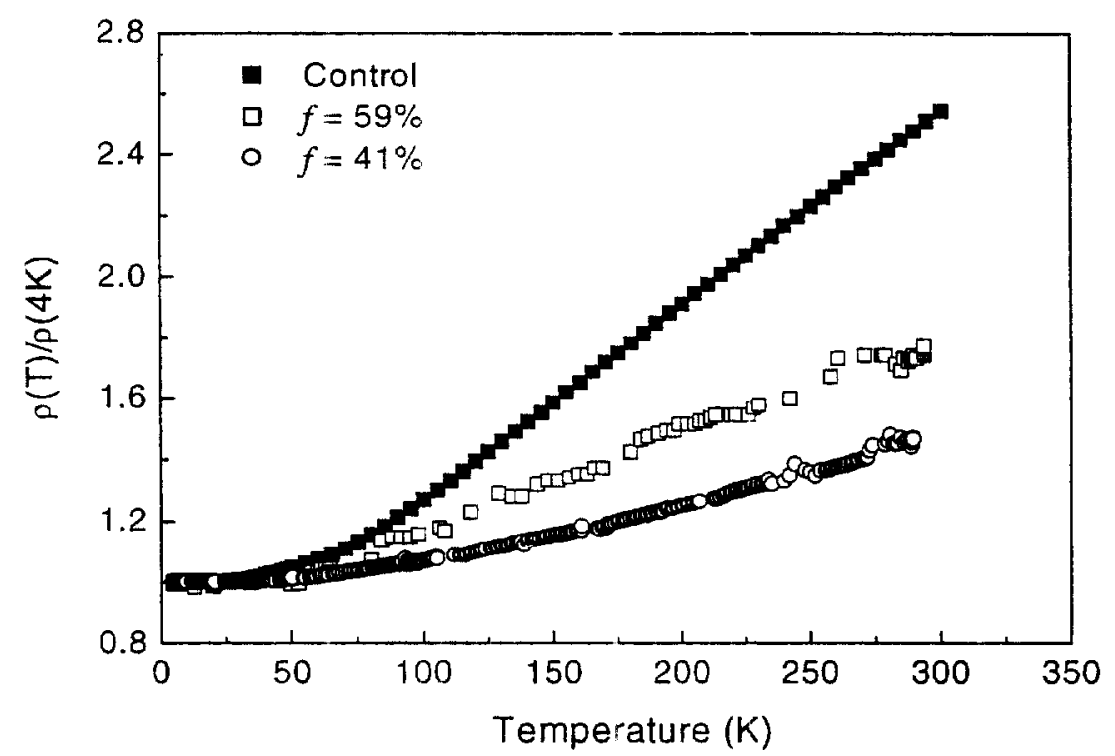

Figure $4 p-T$ of two aluminum anti-dot array strustures with metal fractions, $f$, as indicated. The corresponding data of an aluminum control sample (where $f=100 \%$ ) is also shown for comparison. Note the systematic decrease in the temperature coefficient of the data as $f$ was decreased. 


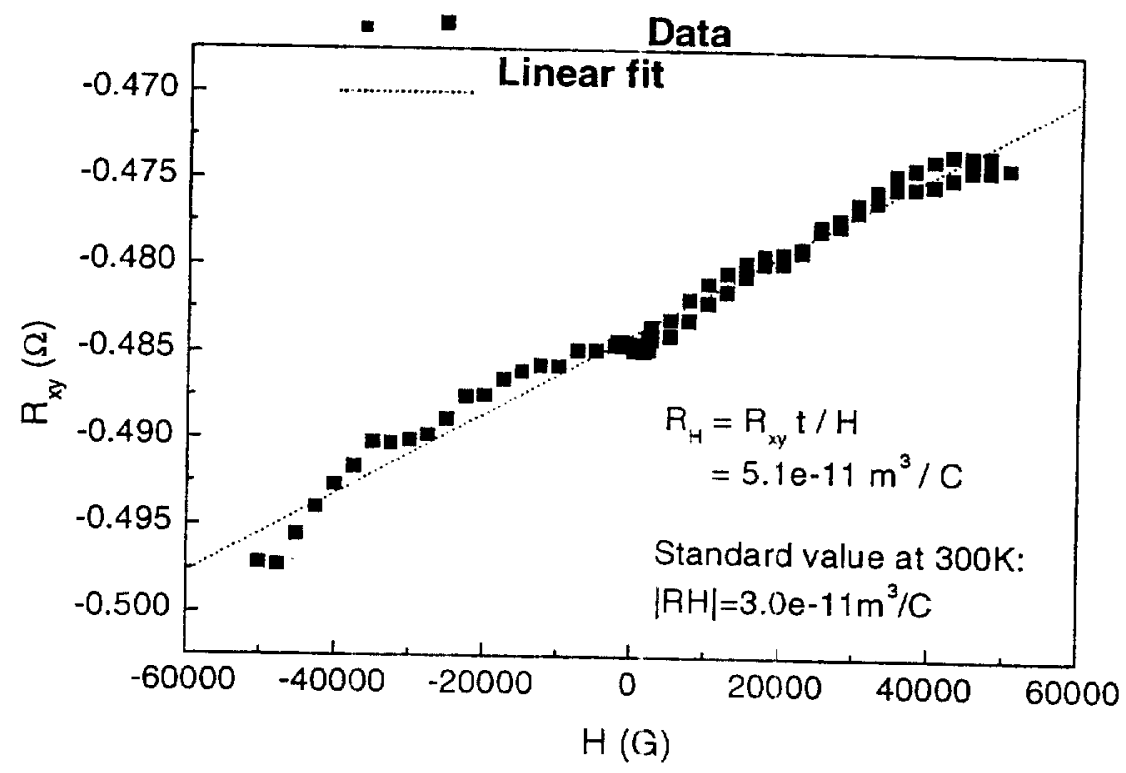

Figure 5 Plot of Hall Resistance versus magnetic $(\mathrm{H})$ field of an aluminum control sample.

\section{CONCLUSION}

In conclusion, we have succeeded in fabricating pre-designed anti-dot arrays with feature sizes $\sim 70 \mathrm{~nm}$ (width) $\times 15 \mathrm{~nm}$ (height) on aluminum using AFM electric field induced oxidation and selective wet etching technique. The demonstration on the fabrication of structures more complex than parallel lines or regular dot arrays are important to show that the AFM oxidation technique is a practical lithographic method. Temperature dependence of electrical resistivity and Hall coefficient obtained at room temperature of an aluminum control sample exhibit good agreement with literature data of aluminum in bulk. Further measurements on the samples containing the anti-dot patterns show that the temperature coefficient decreases systematically with decreasing metal fraction. These results measurements illustrate reliability of the fabrication process we have developed for making pre-designed nanostructures and the contact leads in aluminum by AFM FILO.

\section{ACKNOWLEDGEMENTS}

We are grateful to crucial discussions with Prof. Ping Sheng. Financial support from the Research Grant Council of Hong Kong under project no. HKUST6150/01P is acknowledged.

\section{REFERENCES}

Boisen, A., Birkelund, K., Hansen, O. and Grey, F., 1998, Fabrication of submicron suspended structures by laser and atomic force microscopy lithography on aluminum combined with reactive ion etching, Journal of Vacuum Science and Technology. B, 16, pp. 2977-2981. 
Bouchiat, V. and Esteve, D., 1996, Lift-off lithography using an atomic force microscope, Applied Physics Letters, 69, pp. 3098-3100.

Campbell, P. M., Snow, E. S. and McMarr, P. J., 1995, Fabrication of nanometer-scale side-gated silicon field effect transistors with an atomic force microscope, Applied Physics Letters, 66, pp. 1388-1390.

Chien, F.S.-S., Chang, J.-W., Lin, S.-W., Chou, Y.-C., Chen, T.T., Gwo, S., Chao, T.-S. and Hsieh, W.-F., 2000, Nanometer-scale conversion of $\mathrm{Si}_{3} \mathrm{~N}_{4}$ to $\mathrm{SiO}_{\mathrm{x}}$, Applied Physics Letters, 76, pp. 360-362.

Dagata, J. A., 1995, Device fabrication by scanned probe oxidation, Science, 270, pp. 1625-1626.

Fontaine, P. A., Dubois, E. and Stievenard, D., 1998, Characterization of scanning tunneling microscopy and atomic force microscopy-based techniques for nanolithgraphy on hydrogen-passivated silicon, Joumal of Applied Physics, 84, pp. 1776-1781.

Gwo, S., Yeh, C.-L., Chen, P.-F., Chou, Y.-C., Chen, T. T., Chao, T.-S., Hu, S.-F. and Huang, T.-Y., 1999, Local electric-field-induced oxidation of titanium nitride films, Applied Physics Letters, 74, pp. 1090-1092.

Hu, S., Hamidi, A., Altmeyer, S., Köster, T., Spangenberg, B. and Kurz, H., 1998, Fabrication of silicon and metal nanowires and dots using mechanical atomic force lithography, Journal of Vacuum Science and Technology B, 16, pp. 2822-2824.

Ishibashi, M., Sugita, N., Heike, S., Kajiyama, H. and Hashizume, T., 1999, Fabrication of high-resolution and high-aspect-ratio patterns on a stepped substrate by using scanning probe lithography with a multiplayer-resist system, Japan Journal of Applied Physics 38, pp. 2445-2447.

Itatani, T., Segawa, K., Matsumoto, K., Ishii, M., Nakagawa, T., Ohta, K. and Sugiyama, Y., 1996, Ultrafast Metal-Semiconductor-Metal Photoconductive Switches Fabricated Using an Atomic: Force Microscope, Japan Journal of Applied Physics, 35, pp. 1387-1389.

Minne, S. C., Soh, H. T., Flueckiger, Ph. and Quate, C. F., 1995, Fabrication of $0.1 \mathrm{um}$ metal oxide semiconductor field-effect transistors with the atomic force microscope, Applied Physics Letters, 66, pp. 703-705.

Shirakashi, J., Ishii, M., Matsumoto, K., Miura, N. and Konagai, M., 1996, Surface Modification of Niobium (Nb) by Atomic Force Microscope (AFM) Nano-Oxidation Process, Japan Journal of Applied Physics, 35, pp. 1524-1527.

Snow, E.S., Park, D. and Campbell, P.M., 1996, Single-atom point contact devices fabricated with an atomic force microscope, Applied Physics Letters, 69, pp. 269-271.

Stokes, H. T., 1987, Solid State Physics (Boston: Allyn and Bacon), p. 300.

Tsau, L., Wang D. and Wang, K.L., 1994, Nanometer scale patterning of silicon (100) surfaces by an atomic force microscope operating in air, Applied Physics Letters, 64, pp. 2133-2135.

Zhang, X.X., Liu, H. and Pakhomov, A.B., 2000, Observation of giant Hall effect in non-magnetic cermets, Physic a $B, 279$, pp. 81-93. 